\title{
Analysis of the effect of okra extract on the diversity of intestinal flora in diabetic rats based on 16S rRNA sequence
}

\author{
Li WU ${ }^{1,2,3,4 \#}$ (D), Baosha TANG ${ }^{1,2 \#}$, Pufu LAI ${ }^{1,2}$, Minjie WENG ${ }^{1,2}$, Hengguang ZHENG ${ }^{1,2}$, Junchen CHEN ${ }^{1,2}$, Yibin LI ${ }^{1,2 *}$ (D)
}

\begin{abstract}
To study the effect of okra aqueous extract on the intestinal flora of diabetic rats. Taking normal SD rats as CK group, diabetic rats induced by $40 \mathrm{mg} /(\mathrm{kg} \bullet \mathrm{bw})$ streptozotocin (STZ) intraperitoneally as $\mathrm{M}$ group, normal and diabetic rats gavaged with $500 \mathrm{mg} /(\mathrm{kg} \bullet \mathrm{bw})$ okra aqueous extract were CKO and $\mathrm{MO}$ groups,respectively. The bacterial flora of the cecum contents of rats was analyzed with $16 \mathrm{~S}$ rRNA V3-V4 sequencing after 8 weeks of the experiment. Compared with CK, M increased Firmicutes $14.00 \%$ and decreased Proteobacteria 19.32\% ( $<<0.01$ ). Compared with M, MO down-regulated Lachnospiraceae 2.64\% (such as Blautia, Anaerostipes), up-regulated Peptostreptococcaceae 1.20\% in Firmicutes, and down-regulated Desulfovibrioceae 3.78\% (such as Desulfovibrio) and up-regulated Burkholderia 5.24\% (such as Parasutterella) in Proteobacteria. It is worth noting that MO up-regulated Christensenellaceae (LDA $>4)$, which was negatively correlated with body mass index, inflammation, and metabolic syndrome. The okra aqueous extract reverses the changes of the intestinal flora of STZ-induced diabetic rats, which decreased Firmicutes and increased Proteobacteria by down-regulating Lachnospiraceae and Desulfovibrionaceae, up-regulating Burkholderiaceae, Christensenellaceae. The okra aqueous extract has the potential as a functional food with regulating intestinal microecology balance of diabetes.
\end{abstract}

Keywords: okra; aqueous extract; intestinal flora; diabetes; ameliorate intestinal microecology.

Practical Application: To study the effect of the water extract of okra in regulating the intestinal microecology of diabetic rats, and to provide a research basis for the natural food resources for lowering blood sugar and blood lipids.

\section{Introduction}

In 2019, the statistics of the Food and Agriculture Organization of the United Nations (FAO) show that the global okra production is about 9.95 million tons, and the trade volume is over 5 billion dollars (Food and Agriculture Organization of the United Nations, 2019). At present, it is widely grown in Africa and Asia, and its production share by region about $32.2 \%$ and $66.9 \%$. Especially okra is grown on a large scale in the Fujian and Hainan provinces of China. Through the comprehensive utilization of okra, the problems that the fresh okra fruits are different to preserve and are easily aging and affect the fruit quality are solved (Tang et al., 2017; Weng et al., 2017). Previous studies have confirmed that okra pods are rich in mucus (biological polysaccharides, sugar polymers), flavonoids, and dietary fiber, which have the effect of regulating glucose and lipid metabolism, promoting bowel movement, and preventing constipation (Georgiadis et al., 2011; Wu et al., 2020; Yuan et al., 2019). Diabetes mellitus (DM) is one of the most common diseases caused by metabolic disorders in humans. According to the prediction of the World Health Organization (WHO) by 2030, the number of diabetes may be about 439 million (Shaw et al., 2010). Among them, approximately $10 \%$ of clinical diabetes patients are insulin-dependent diabetes (IDDM), that is, type 1 diabetes (T1DM) (Gero, 2010; Lee et al., 2010; O'Callaghan, 2017). Abnormal blood glucose metabolism and abnormal lipid metabolism usually occur together (Guo et al., 2019; Lv et al., 2019; Li et al., 2019). In recent years, the regulation of glucose and lipid metabolic disorders has become a focus of research (Hu et al., 2018). The young, old and second fruits (including pod and pedicle) of okra have low commercial value, but they are rich in water-soluble components such as biological polysaccharides. Therefore, using them as the raw material for hot water extraction, the okra aqueous extract was prepared. Based on the scientific analysis of the active ingredients of okra aqueous extract and the molecular weight distribution of polysaccharides, the quantitative evaluation of the efficacy of regulating the intestinal flora of diabetes was carried out, thereby expanding the application of okra in the field of functional food. To investigated the effect of okra aqueous extract on the diversity of intestinal flora in diabetic rats, the metagenomic DNA of cecum content of twelve rats was extracted, then the 16SrRNA V3-V4 region of the flora was sequenced by the latest IonS5 ${ }^{\mathrm{TM}} \mathrm{XL}$ SE400/ SE600 sequencing technology.

\footnotetext{
Received 02 Jan., 2021

Accepted 09 Feb., 2021

${ }^{1}$ Institute of Agricultural Engineering Technology, Fujian Academy of Agricultural Sciences, Fuzhou, Fujian Province, China

${ }^{2}$ Fujian Key Laboratory of Agricultural Product (Food) Processing, Fuzhou, Fujian Province, China

${ }^{3}$ College of Chemistry, Fuzhou University, Fuzhou, Fujian Province, China

${ }^{4}$ Institute of Food Science and Technology, College of Biological Science and Technology, Fuzhou University, Fuzhou, Fujian, P. R. China

"co-first authors: Li Wu and Baosha TANG contributed equally to this study.

*Corresponding author: xxj1963@163.com, lyb9951@163.com
} 


\section{Materials and methods}

\subsection{The okra aqueous extract and animal experiment}

The method of preparation of okra aqueous extract and animal experiment according to the reference (Wu et al., 2020). Dried okra was extracted with distilled water at 1:25 (W/V). The okra mixture was heated at $100{ }^{\circ} \mathrm{C}$ for 2 hours, and then filtered with two layers of gauze. The filtrate was centrifuged at $3500 \mathrm{rpm}$ for 10 minutes to separate the supernatant. The supernatant was concentrated to $1 / 3-1 / 4$ volume by a rotary evaporator at $60{ }^{\circ} \mathrm{C}, 30 \mathrm{rpm}$. The concentrated liquid was freeze-dried by a freeze dryer. The okra aqueous extract was dissolved in sterile distilled water. After 8 weeks of gavage, the cecal contents of rats of four groups were collected for determination of intestinal flora. Taking normal SD rats as CK group, diabetic rats induced by $40 \mathrm{mg} /(\mathrm{kg} \bullet \mathrm{bw}) \mathrm{STZ}$ intraperitoneally as M group, normal and diabetic rats gavaged with $500 \mathrm{mg} /(\mathrm{kg} \bullet \mathrm{bw})$ okra aqueous extract were $\mathrm{CKO}$ and $\mathrm{MO}$ groups,respectively.

\subsection{Determination of intestinal flora}

The genomic DNA from the cecum content of 12 rats was extracted and electrophoresed with $1 \%$ agarose gel (Zhou et al., 2019). The V3-V4 region of the bacterial 16S rRNA gene was amplified using 341F ( $5^{\prime}$-CCT AYG GGR BGC ASC AG-3') and 806R ( $5^{\prime}$-GGA CTA CNN GGG TAT CTA AT-3'). The sequencing and bioinformatics showed that the analysis on IonS5TMXL sequencing platform (Thermofisher) generated 415 bp DNA fragment, which was completed by Beijing Nuohe Zhiyuan Technology Co., Ltd. (Shanghai, China)

The raw reads were quality processed by Usearch software to remove low-quality reads, remove reads containing adapters, and remove reads containing too much $\mathrm{N}$. Then, the sequence reads were classified into operational taxonomic units (OTUs) by UPARSE (version 7.0.1001) with a $97 \%$ similarity cut-off. The obtained sequence in the SSU rRNA database (http://www.arb-silva.de/) was compared and species annotated by the method of Mothur and SILVA132 at the threshold $0.8 \sim 1$. The taxonomy analysis and heatmap were performed on the Novomagic cloud computing platform. The taxonomic information was obtained and the community composition of each sample was counted at each taxonomic level, including kingdom, phylum, class, order, family, genus, and species. PCA score was drawn using the SIMCA-14.1 (UMETRICS, Sweden) based on the relative abundance of gut microbiota at the genus level. The T-test was analyzed based on R 2.15.3 software.

\subsection{Statistical analysis}

The data processing of offline sequencing, OTU clustering, and species annotation, sample complexity analysis (alpha diversity), beta diversity analysis (beta diversity), and correlation analysis of environmental factors were commissioned by Beijing Nuohe Zhiyuan Technology Co., Ltd.

\section{Results and discussion}

\subsection{DNA extraction and quality inspection of cecum contents of rats}

The result of agarose gel electrophoresis shown obvious DNA bands. The DNA concentration was measured by the ScanDrop 200 ultra-micro ultraviolet spectrophotometer (Jena Company, Germany). The DNA concentration was over $200 \mathrm{ng} / \mu \mathrm{L}$, and the OD260/280 value was 1.8-2.0. Then, the extracted intestinal microbiota genomic was used as a template for PCR amplification with bacterial 16S rRNA gene universal primer 341F/806R. After amplification, the size of the target DNA fragment was about $415 \mathrm{bp}$, further indicating the extracted genomic meets the quality requirements of IonS5 ${ }^{\mathrm{TM}} \mathrm{XL}$ SE400/SE600 highthroughput sequencing.

\subsection{Sequencing sequence quality analysis}

According to the quality control standard of RNA sequence, the result (Table 1) showed that the total number of clean reads was 810507 , of which the lowest sequence number was

Table 1. Statistics of sample sequencing data.

\begin{tabular}{|c|c|c|c|c|c|}
\hline Sample & Sequencing area & Raw reads & Clean reads & $\begin{array}{c}\text { Average sequence } \\
\text { length (bp) }\end{array}$ & Effective(\%) \\
\hline CK2 & V3-V4 & 77060 & 73476 & 417 & 95.35 \\
\hline M1 & $\mathrm{V} 3-\mathrm{V} 4$ & 87047 & 80166 & 414 & 92.10 \\
\hline M2 & V3-V4 & 84342 & 80277 & 415 & 95.18 \\
\hline $\mathrm{CKO} 2$ & V3-V4 & 55389 & 50203 & 417 & 90.64 \\
\hline $\mathrm{CKO} 3$ & $\mathrm{~V} 3-\mathrm{V} 4$ & 65528 & 62735 & 418 & 95.74 \\
\hline MO1 & V3-V4 & 85314 & 80096 & 416 & 93.88 \\
\hline MO2 & V3-V4 & 66531 & 59936 & 411 & 90.09 \\
\hline MO3 & V3-V4 & 89009 & 80276 & 415 & 90.19 \\
\hline
\end{tabular}

Note: CK group: Normal rats; CKO group: Normal rats treated with okra aqueous extract; M group: Diabetic rats; MO group: Diabetic rats treated with okra aqueous extract. 
CKO3 (50203) and the highest number was M2 (80277). Using GeneiousV4.8 to count the length of the average sequence and the percentage of the number of clean reads to the number of raw reads (Effective), the results show that the average sequence length was about $415.4 \mathrm{bp}$, which was in line with the expected fragment size. The effectiveness was over $91.05 \%$

\subsection{Analysis of the diversity of rat intestinal flora based on community structure identification and construction system}

\section{Species annotation and cluster analysis of intestinal flora}

The results were displayed that a total number of OTUs was 909 , which obtained Firmicutes 631 OTUs, Bacteroidetes 128 OTUs, and Proteobacteria 27 OTUs, and the identified bacterial flora belonged to 11 families, 17 classes, 23 orders, 42 families, and 92 genera.

Relative abundance in phylum level showed as Figure 1A. Firmicutes, Bacteroidetes, and Proteobacteria were three main bacterial phyla, accounting for $97.74 \%$ 99.28\% of the total number of RNA sequencing. Compared with the intestinal flora of normal rats $(\mathrm{CK}, \mathrm{CKO})$, the proportion of Firmicutes of diabetic rats $(\mathrm{M}, \mathrm{MO})$ accounted for about $70.00 \%$, which was nearly $14.00 \%$ higher than $56.00 \%$ of normal rats $(P<0.01)$, while the proportion of Proteobacteria in diabetic rats $(\mathrm{M}, \mathrm{MO})$ was $5.30 \%$, which was $19.32 \%$ lower than $24.62 \%$ in normal rats $(P<0.01)$. The okra aqueous extract treatment group (MO) tends to reduce the proportion of Firmicutes and increase the proportion of Proteobacteria. It was reported in other literature that streptozotocin-induced changes in the structure of the intestinal flora of diabetic rats (Yuan et al.,
2018) at the phylum level. The relative abundance of the Firmicutes and Bacteroidetes were increased, while the relative abundance of the Proteobacteria was decreased. The ratio of Firmicutes/ Bacteroidetes decreased (Huang et al., 2018; Lv et al., 2019). It was consistent with the results of this paper.

Relative abundance in the family level of Firmicutes, Bacteroides, and Proteobacteria exhibited in Figure 1B, and Table 2. There were 18 bacteria families in Firmicutes phylum were identified, including Ruminococcaceae 50.99\%, Lachnospiraceae 28.25\%, Lactobacillaceae 8.19\%, Peptostreptococcaceae 5.24\%, and Christensenellaceae $1.58 \%$. There were 7 bacteria families in the Bacteroides phylum were identified, including Prevotellaceae 24.57\%, Muribaculaceae $66.05 \%$, Bacteroidaceae $6.66 \%$, and Rikenellaceae $1.47 \%$. There were 4 bacteria families in the Proteobacteria phylum were identified, including Desulfovibrionaceae 92.59\%, Burkholderiaceae 6.27\%, Enterobacteriaceae $0.95 \%$, and Moraxellaceae $0.03 \%$.

Comparison of the rate of the sequence number of main families between normal rats groups and diabetic rats groups (CK vs. $\mathrm{M}, \mathrm{CKO}$ vs. $\mathrm{MO}$ ), and between non-okra aqueous extract treatment groups and okra aqueous extract treatment groups (CK vs. CKO, M vs. MO). In the phylum of Firmicutes (Table 2), the Lachnospiraceae family of diabetic rats groups was upregulated $1.46 \%$ and $4.98 \%$, while the Peptostreptococcaceae family was decreased by $4.68 \%$ and $7.60 \%$, respectively. It is worth noting that the changes of Lachnospiraceae and Peptostreptococcaceae in normal rats and diabetic rats treated with okra aqueous extract were reversed, for instance, Lachnospiraceae of CKO and MO were decreased $6.16 \%$ and $2.64 \%$, respectively, while the
A
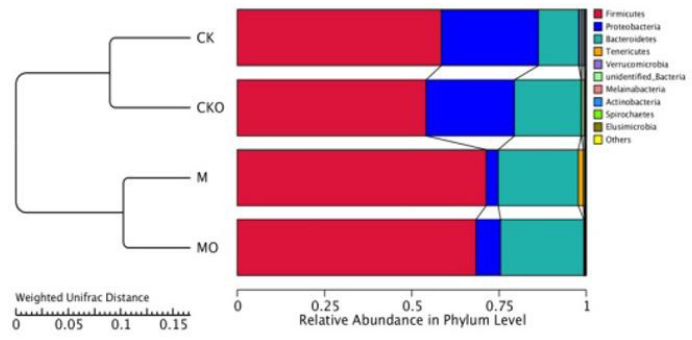

$\mathrm{C}$

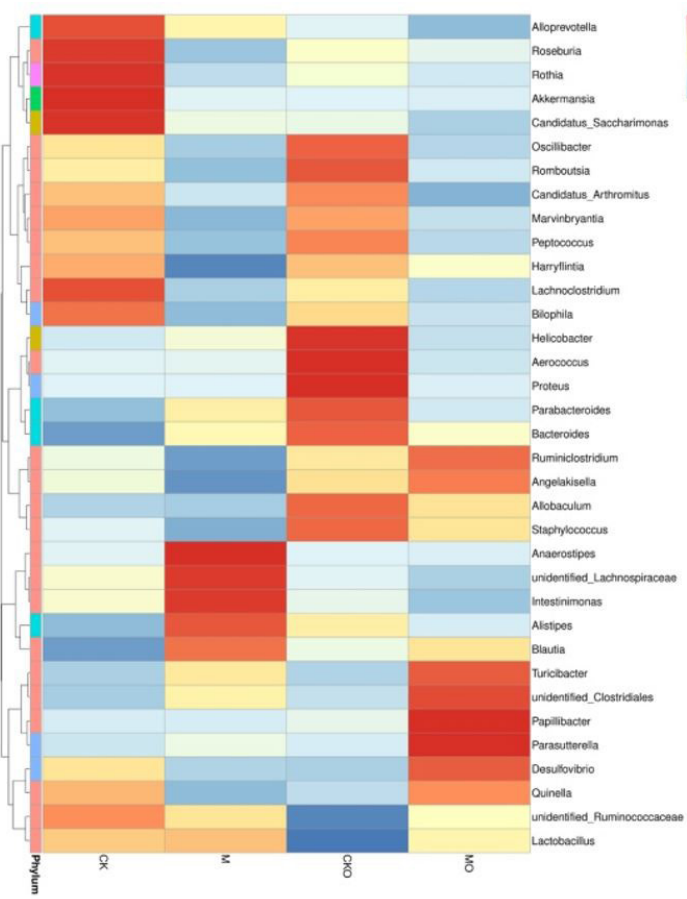

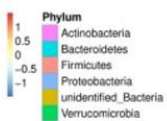
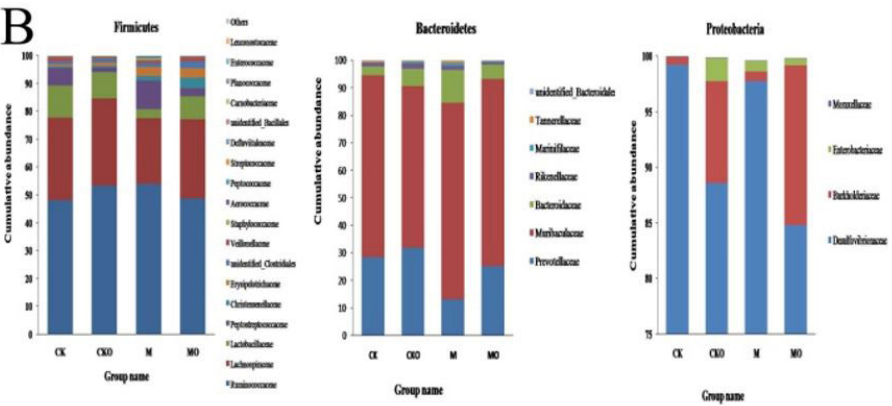

Figure 1. Species annotation and relative abundance of the intestinal flora of rats in all groups. (A) Relative abundance in phylum level; (B) Relative abundance in the family level of Firmicutes, Bacteroides, and Proteobacteria; (C) Heat map of relative abundance in genus level. CK group: Normal rats; CKO group: Normal rats treated with okra aqueous extract; M group: Diabetic rats; MO group: Diabetic rats treated with okra aqueous extract. 
Table 2. Comparison of the rate of the sequence number of intestinal flora at the family-level of Firmicutes, Bacteroides, and Proteobacteria (\%).

\begin{tabular}{|c|c|c|c|c|c|c|c|c|c|}
\hline Phylum & Family & CK & $\mathrm{CKO}$ & M & $\mathrm{MO}$ & CK vs. M & $\begin{array}{c}\text { CKO vs. } \\
\text { MO }\end{array}$ & $\begin{array}{l}\text { CK vs. } \\
\text { CKO }\end{array}$ & $\mathrm{M}$ vs. MO \\
\hline \multirow{5}{*}{ Firmicutes } & Ruminococcaceae & 48.05 & 53.92 & 53.33 & 48.65 & -5.28 & 5.27 & -5.87 & 4.68 \\
\hline & Lachnospiraceae & 29.72 & 23.56 & 31.18 & 28.55 & -1.46 & -4.98 & 6.16 & 2.64 \\
\hline & Lactobacillaceae & 11.54 & 3.31 & 9.60 & 8.32 & 1.94 & -5.00 & 8.23 & 1.29 \\
\hline & Peptostreptococcaceae & 6.25 & 10.36 & 1.57 & 2.77 & 4.68 & 7.60 & -4.11 & -1.20 \\
\hline & Christensenellaceae & 0.48 & 1.47 & 0.62 & 3.74 & -0.14 & -2.27 & -0.98 & -3.11 \\
\hline \multirow{4}{*}{ Bacteroidetes } & Prevotellaceae & 28.33 & 13.08 & 31.72 & 25.15 & -3.38 & -12.07 & 15.26 & 6.57 \\
\hline & Muribaculaceae & 66.04 & 71.40 & 58.87 & 67.90 & 7.17 & 3.50 & -5.36 & -9.03 \\
\hline & Bacteroidaceae & 3.41 & 11.80 & 6.14 & 5.27 & -2.73 & 6.53 & -8.39 & 0.87 \\
\hline & Rikenellaceae & 1.18 & 1.71 & 2.08 & 0.90 & -0.89 & 0.80 & -0.52 & 1.17 \\
\hline \multirow{4}{*}{ Proteobacteria } & Desulfovibrionaceae & 99.20 & 97.74 & 88.59 & 84.81 & 10.61 & 12.94 & 1.45 & 3.78 \\
\hline & Burkholderiaceae & 0.71 & 0.86 & 9.13 & 14.37 & -8.42 & -13.51 & -0.15 & -5.24 \\
\hline & Enterobacteriaceae & 0.08 & 0.97 & 2.13 & 0.60 & -2.05 & 0.37 & -0.90 & 1.53 \\
\hline & Moraxellaceae & 0.02 & 0.02 & 0.05 & 0.04 & -0.03 & -0.02 & 0.00 & 0.01 \\
\hline
\end{tabular}

Note: CK group: Normal rats; CKO group: Normal rats treated with okra aqueous extract; M group: Diabetic rats; MO group: Diabetic rats treated with okra aqueous extract. Rate $>0.05 \%$.

Peptostreptococcaceae of CKO and MO were increased $4.11 \%$ and $1.20 \%$, respectively. In the phylum of Bacteroidetes, the Prevotellaceae of diabetic rats groups were upregulated 3.38\% and $12.07 \%$, while the Muribaculaceae were decreased by $7.17 \%$ and $3.50 \%$, respectively. After the treatment with okra aqueous extract, Prevotellaceae were decreased by $15.26 \%$ and $6.57 \%$, respectively, while the Muribaculaceae was increased by $5.36 \%$ and $9.03 \%$. In the phylum of Proteobacteria, the Desulfovibrionaceae of diabetic rats groups was downregulated $10.61 \%$ and $12.94 \%$, while the Burkholderiaceae was increased by $8.42 \%$ and $13.51 \%$, respectively. After the okra aqueous extract treatment, the changes become smaller, for example, Desulfovibrionaceae was decreased by $1.45 \%$ and $3.78 \%$, while the Burkholderiaceae was increased $0.15 \%$ and $5.24 \%$, respectively.

In conclusion, the treatment of okra aqueous extract significantly repaired the changes in intestinal flora caused by diabetes. Diabetes up-regulated Lachnospiraceae 4.98\%, Prevotellaceae 12.07\%, and Burkholderiaceae 13.51\%, downregulated Peptostreptococcaceae 7.60\%, Muribaculaceae 3.50\%, and Desulfovibrionaceae $12.94 \%$. The okra aqueous extract down-regulates Lachnospiraceae 2.64\%, Prevotellaceae 6.57\%, Desulfovibrionaceae $3.78 \%$, and up-regulates Peptostreptococcaceae 1.20\% Muribaculaceae 9.03\%, and Burkholderiaceae 5.24\%.

Figure $2 \mathrm{C}$ showed the heat map of relative abundance in the genus level. Compared with the CK group, the M group significantly upregulated three genera of Firmicutes phylum, involving Anaerostipes, unidentified_Lachnospiraceae, Intestinimonas. Compared with the M group, the MO group treated with okra extract could downregulate the above bacterial genera.

\section{Analysis of alpha diversity index of the complexity of intestinal flora of rats}

From the Venn diagram (Figure 3A), there were 490 OTUs in common among four groups, and 13,5,28,14 kinds of unique OTUs in $\mathrm{CK}, \mathrm{CKO}, \mathrm{M}$, and $\mathrm{MO}$, respectively.
Shannon index and Simpson index described community diversity, including species richness and uniformity (Figure 3B). The larger the Shannon index and Simpson index, the higher the diversity of the sample community. The order of Shannon index and Simpson index from big to small were as follows: $\mathrm{M}>\mathrm{MO}>\mathrm{CKO}>\mathrm{CK}$, indicating that the bacterial flora of diabetic rats was more diverse than that of normal rats. The diversity of the bacterial flora of rats treated with okra extract was between normal rats and diabetic rats.

Figure $3 \mathrm{C}$ showed the dilution curve and rank-abundance curve. As the number of sequencing increased to 40000 , the dilution curve tended to be flat, indicating that the number of samples was reasonable and the sequencing depth had covered all species in the sample.

\section{Analysis of beta diversity index of the complexity of intestinal flora of rats}

The beta diversity index of the intestinal flora of rats (Figure 3) showed that the normal rats in the $\mathrm{CK}$ and $\mathrm{CKO}$ group were on the left side of the $\mathrm{Y}$-axis, while the diabetic rats in the $\mathrm{M}$ and $\mathrm{MO}$ group were on the right side of the Y-axis. The distance between CK and CKO group (or M and MO group) was close, indicating that the community composition was similar. Okra aqueous extract had little effect on the intestinal flora of normal rats from the comparison between $\mathrm{CK}$ and $\mathrm{CKO}$ group. However, it had an adjusted effect on the intestinal flora of diabetic rats from the comparison between the $\mathrm{M}$ and $\mathrm{MO}$ group.

\section{Analysis of different species between groups}

Species with significant differences between groups, called biomarkers, were investigated by Lefse analysis (Figure 4A, 4B). The different bacteria with an LDA score greater than 4 in the $\mathrm{MO}$ group was $\mathrm{f}$ _Christensenellaceae, belonging to the Firmicutes phylum (Sánchez-Tapia et al., 2019), which was significantly negatively related to metabolic diseases such as body mass index (BMI) (Waters \& Ley, 2019), inflammation 
A

\section{B}
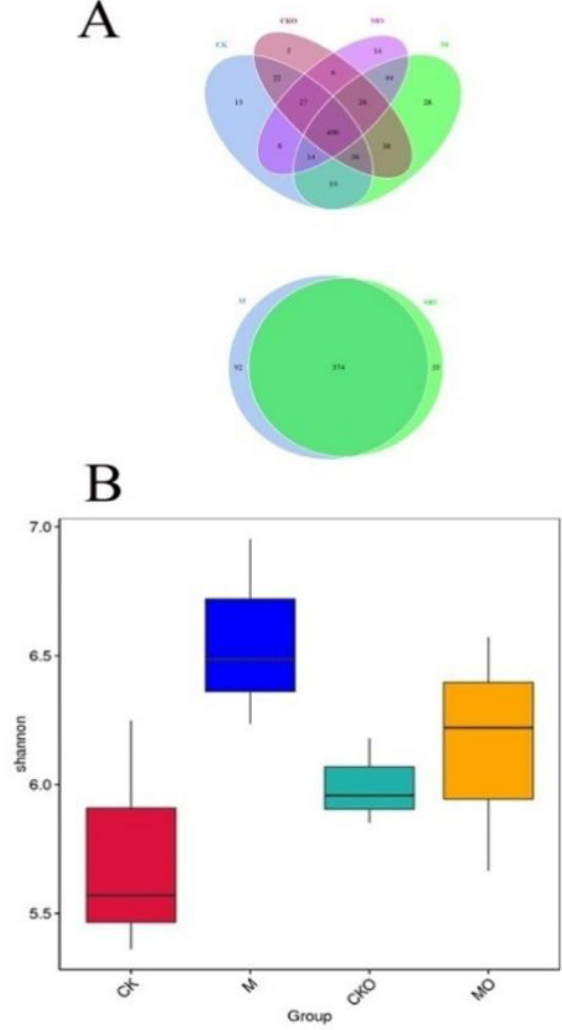
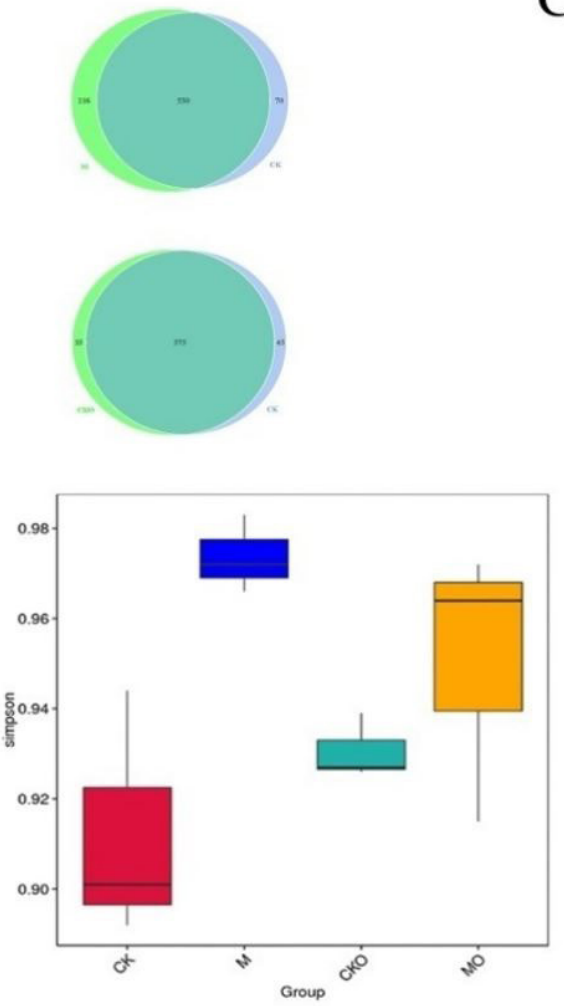
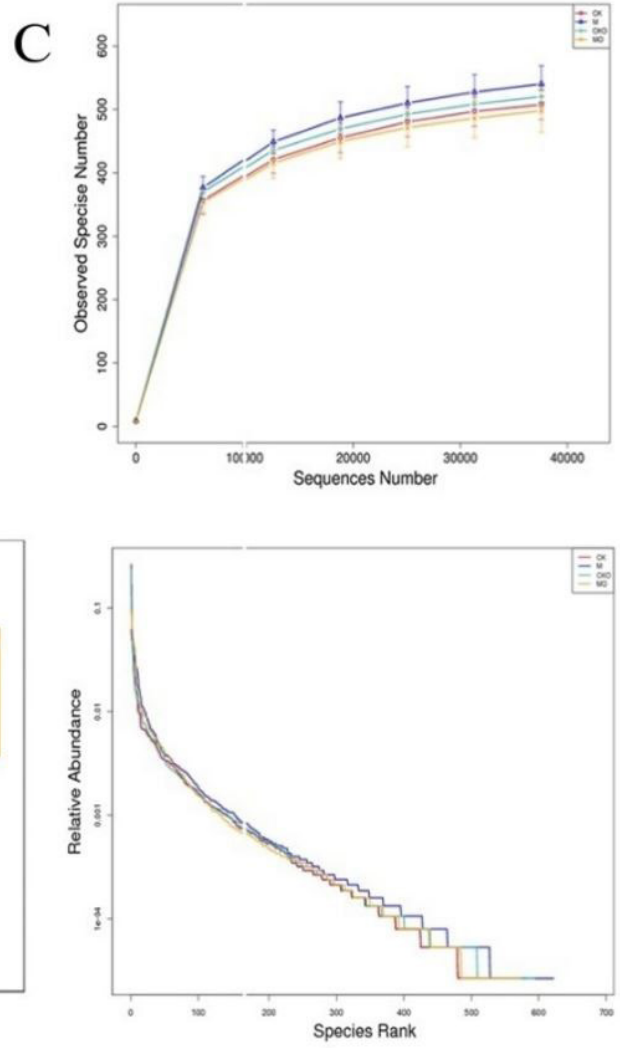

Figure 2. Alpha diversity index of the intestinal flora of rats. (A) Venn diagram; (B) Box plot alpha diversity (Shannon index and Simpson index); (C) Dilution curve and rank-abundance curve. CK group: Normal rats; CKO group: Normal rats treated with okra aqueous extract; M group: Diabetic rats; MO group: Diabetic rats treated with okra aqueous extract.
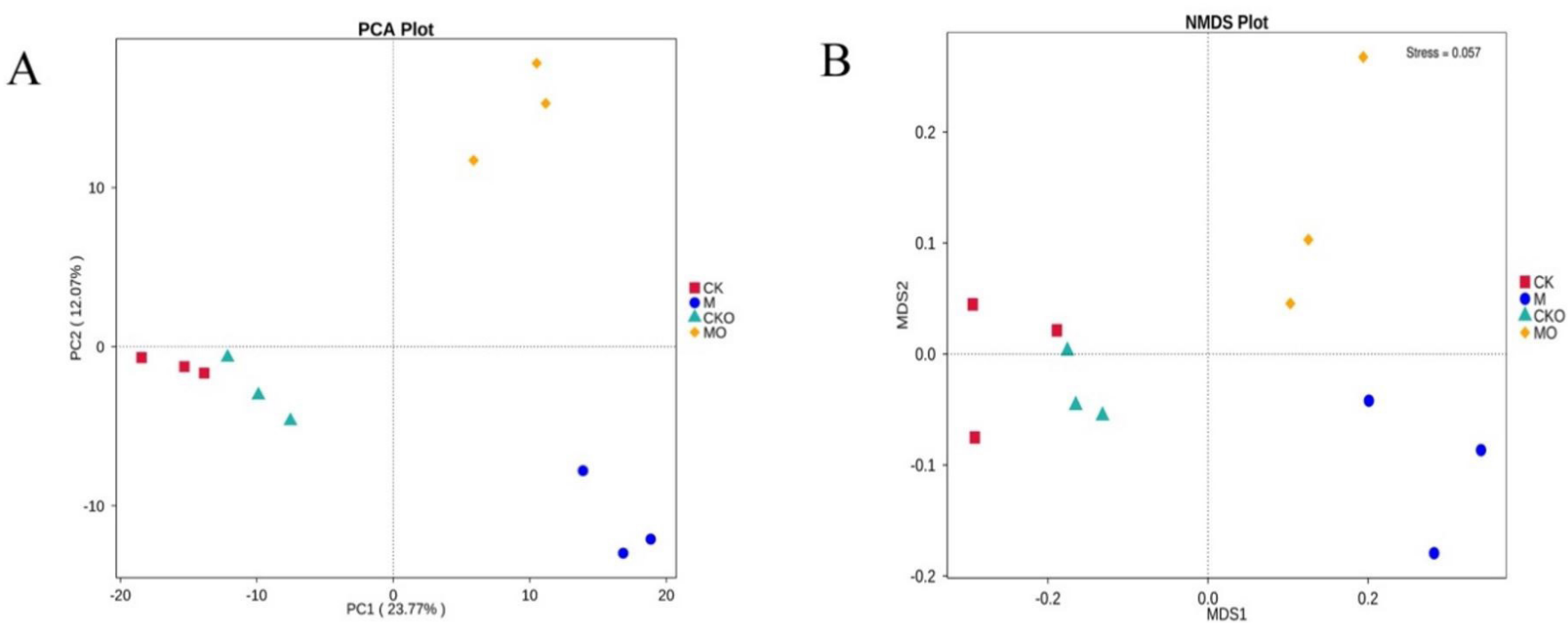

Figure 3. The beta diversity index of the intestinal flora of rats. (A) PCA; (B) NMDS. CK group: Normal rats; CKO group: Normal rats treated with okra aqueous extract; $M$ group: Diabetic rats; MO group: Diabetic rats treated with okra aqueous extract.

and metabolic syndrome. Sichuan Agricultural University reported an increase in the diversity of intestinal microflora and the number of beneficial bacteria in long-lived populations, such as Clostridium, Ruminococcaceae, Akkermansia, and Christensenellaceae (Biagi et al., 2016). The different species in the M group were s_Christen Clostridium_sp_KNHs209 and g_Anaerostipes, and the different species in the CK group were p_Proteobacteria, c_Deltaproteobacteria, o_Desulfovibrionales, f_Desulfovibrionaceae. Compared with the CK group (Figure 5C, 5D), the M group down-regulated Firmicutes_bacterium_ASF500 and 
A

Cladogram

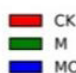

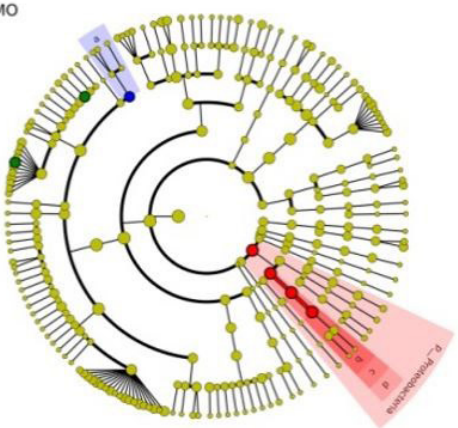

$\mathrm{C}$
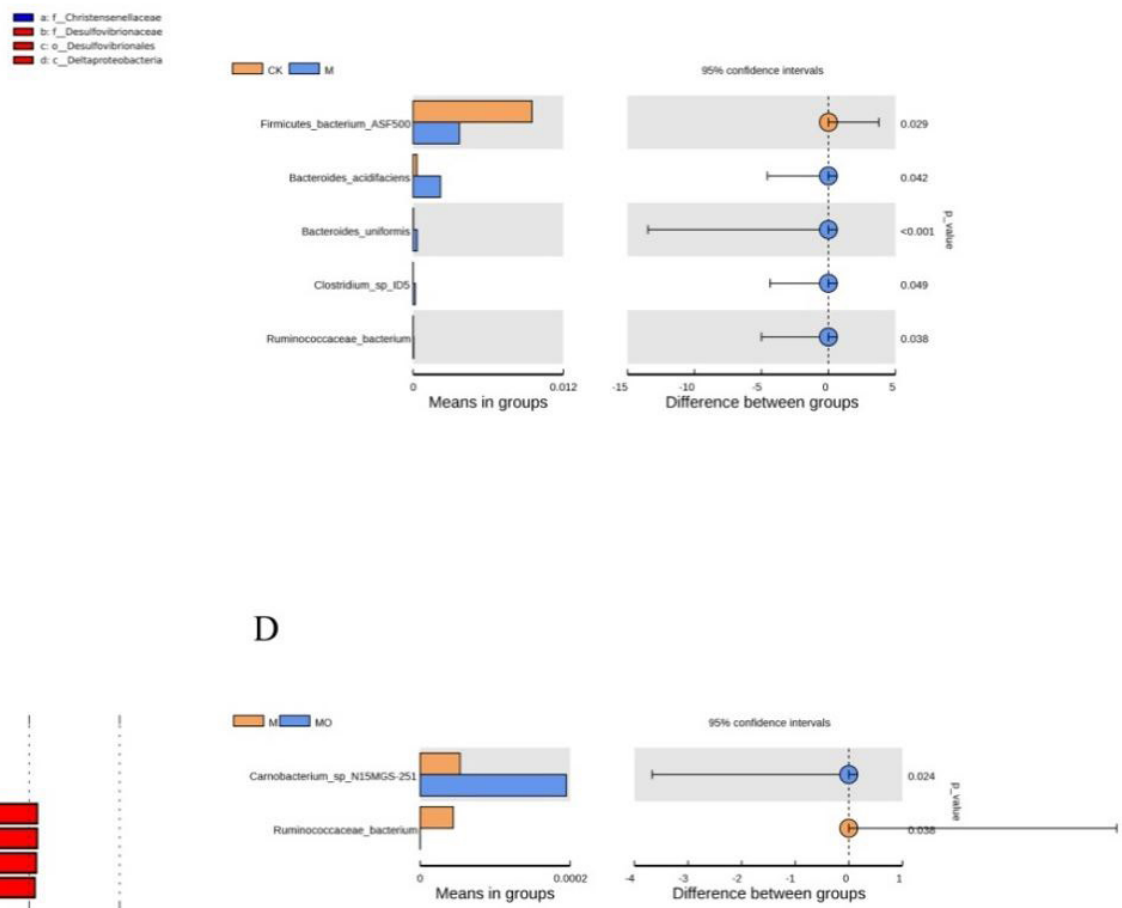

D
B

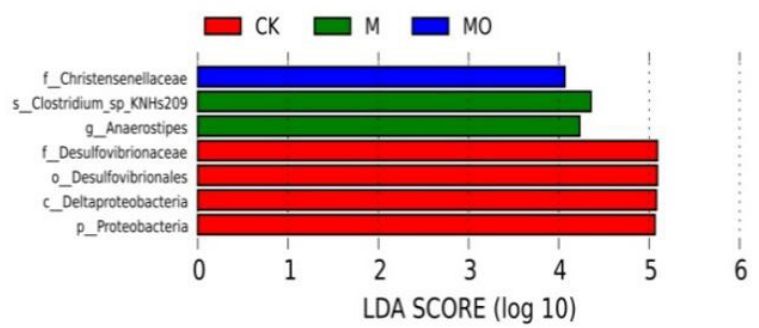

Figure 4. Analysis of the differential intestinal flora between groups (A) LDA tree (B) LDA, (LDA>4) (C) T-test between CK and M group (D). T-test between $\mathrm{M}$ and $\mathrm{MO}$ group Note: The differences between groups were determined using a Welsh's t-test, and the Benjamini-Hochberg procedure was used to control the false-discovery rate due to multiple testing. Corrected $\mathrm{P}$ values are shown at the right. $(P<0.05)$, confidence intervals $=95 \%$. CK group: Normal rats; M group: Diabetic rats; MO group: Diabetic rats treated with okra aqueous extract.

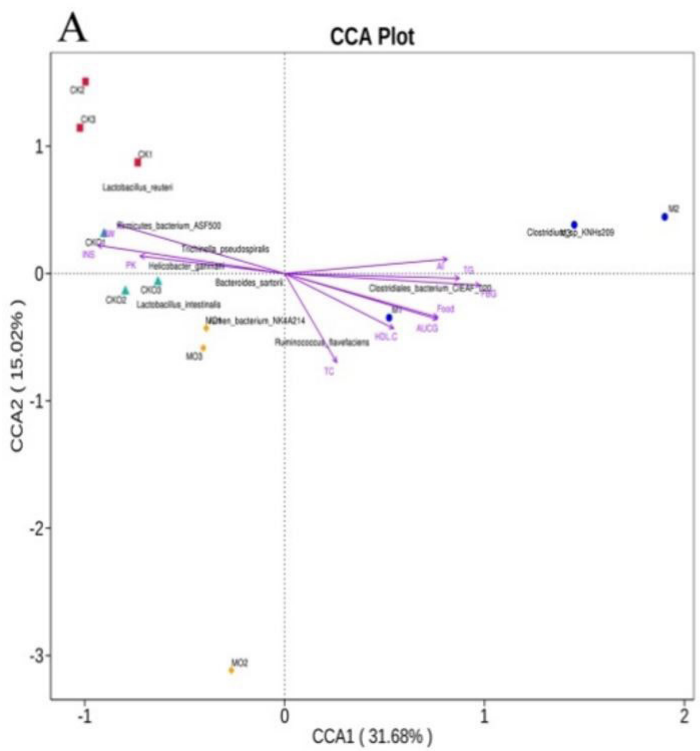

B

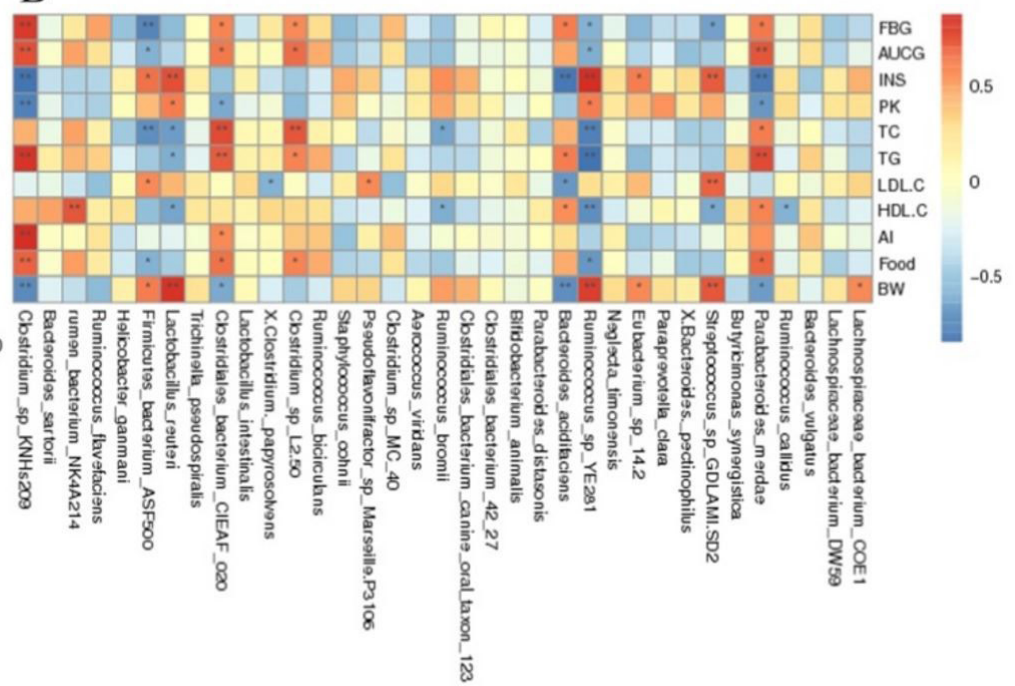

Figure 5. Environmental factor association analysis. (A) CCA; (B) The correlation analysis between the top 35 bacteria genera and environmental factors. CK group: Normal rats; CKO group: Normal rats treated with okra aqueous extract; M group: Diabetic rats; MO group: Diabetic rats treated with okra aqueous extract. 
up-regulated Bacteroides_acidifaciens, Bacteroides_uniformis, Clostridium_sp_ID5, and Ruminococcaceae_bacterium significantly. Compared with the $\mathrm{M}$ group, the MO group upregulated Carnobacterium_sp_N15MGS-251 and down-regulated Ruminococcaceae_bacterium significantly.

\section{Environmental factor association analysis}

If the included angle between environmental factors was an acute/obtuse angle, it indicated a positive/negative correlation between them. Environmental factor association analysis (Figure 5A) could be seen that fasting blood glucose (FBG), area under the curve of glucose (AUCG), total cholesterol (TC), total triglyceride (TG), low-density lipoprotein (LDL-C), and food were positively correlated with the diabetic rats, while insulin (INS), pyruvate kinase (PK), high-density lipoprotein (HDL-C), and body weight (BW) were negatively correlated with the diabetic rats. The longer the radiation of the environmental factor was, the greater the influence degree of the environmental factor was. INS and FBG were the greatest important environmental factors. Diabetic rats were often accompanied with glucose and lipid disorders, so FBG, AUCG, TC, TG, and LDL-C were positively correlated with diabetes, while INS, $\mathrm{PK}$, and BW were negatively correlated with diabetes. It was completely consistent with the correlation analysis result of Figure 5A.

The correlation analysis between the top 35 bacteria genera and environmental factors (Figure 5B). It could be seen that there were positively correlated with diabetic indexes (FBG, AUGG, TC, TG, HDL-C, Food) and Clostridium_sp_KNHs209, Bacteroides_acidifaciens, Clostridiales_bacterium_CIEAF_020, Parabacteroides_merdae, indicating that these bacteria cause diabetes. On the contrary, there were negatively correlated with diabetic indexes (FBG, AUGG, TC, TG, HDL-C, Food) and Firmicutes_bacterium_ASF500,Streptococcus_sp_GDLAMI.SD2, Ruminococcus_sp_YE281, indicating that these bacteria were anti-diabetes. Metagenomics and metabonomics studies found that the hypoglycaemic effect of berberine was mediated the activity of intestinal farnesoid X receptor (FXR) by inhibiting the biotransformation of deoxycholic acid (DCA) by Ruminococcus bromii (Zhang et al., 2020). California Stanford University School of Medicine (Sinha et al., 2020) found that Ruminococcaceae converting primary bile acids into secondary bile acids, which played a key role in the treatment of ulcerative colitis. Research of Grifola frondosa polysaccharide on a high-fat diet and streptozotocin-induced hypoglycemic and hypolipidemic activity in diabetic mice and its relationship with the regulation of intestinal flora (Guo et al., 2020), Streptococcus exhibited a negative correlation with cecal SCFAs (acetic acid, propionic acid, and butyric acid) levels, but positively correlated with serum and liver lipid biochemical indexes.

\section{Conclusion}

In summary, the treatment of okra aqueous extract significantly repaired the changes of intestinal microflora caused by diabetes from different phylum, family, and genus levels. (1) At the phylum level, the Firmicutes phylum of diabetic rats was $15 \%$ higher than that of normal rats, and Proteobacteria were $20 \%$ lower than normal rats $(P<0.05)$;
There was a tendency of decreasing Firmicutes and increasing Proteobacteria in the okra aqueous extract treatment groups; (2) At the family level, diabetes up-regulated Lachnospiraceae 4.98\%, Prevotellaceae $12.07 \%$, and Burkholderiaceae $13.51 \%$, down-regulated Peptostreptococcaceae 7.60\%, Muribaculaceae $3.50 \%$, and Desulfovibrionaceae $12.94 \%$. The okra aqueous extract down-regulates Lachnospiraceae 2.64\%, Prevotellaceae $6.57 \%$, Desulfovibrionaceae $3.78 \%$, and up-regulates Peptostreptococcaceae $1.20 \%$ Muribaculaceae $9.03 \%$, and Burkholderiaceae 5.24\%; (3) In the genus level, diabetic rats significantly upregulated the relative abundance of Clostridium sp_KNHs209, Bacteroides_acidifaciens, Clostridiales_bacterium_ CIEAF_020, and Parabacteroides_merdae. On the contrary, the MO group could up-regulate Firmicutes_bacterium_ASF500, Streptococcus_sp_GDLAMI.SD2 and Ruminococcus_sp_YE281 significantly; (4) The different bacteria in the okra aqueous extract group was Christensenellaceae, which was significantly negatively correlated with obesity, inflammation, metabolic syndrome, and was beneficial to human health. The okra aqueous extract reverses the changes of the intestinal flora of STZ-induced diabetic rats, which decreased Firmicutes and increased Proteobacteria by down-regulating Lachnospiraceae and Desulfovibrionaceae, up-regulating Burkholderiaceae, Christensenellaceae. The okra aqueous extract has the potential as a functional food with regulating intestinal microecology balance of diabetes.

\section{Acknowledgements}

This work was supported by funding from the National Key R\&D Program of China [2018YFD0400203], Fujian provincial department of science and technology, China [2020R1032003, 2020R1032004, 2018R1014-6, 2019R1032-12, and 2019J01116], and Fujian Academy of Agricultural Sciences [CXPT202006].

\section{References}

Biagi, E., Franceschi, C., Rampelli, S., Severgnini, M., Ostan, R., Turroni, S., Consolandi, C., Quercia, S., Scurti, M., Monti, D., Capri, M., Brigidi, P., \& Candela, M. (2016). Gut microbiota and extreme longevity. Current Biology, 26(11), 1480-1485. http://dx.doi.org/10.1016/j. cub.2016.04.016. PMid:27185560.

Food and Agriculture Organization of the United Nations - FAO. (2019). Rome, Italy: FAO. Retrieved from http://fao.org/faostat/ en/\#data/QC.

Georgiadis, N., Ritzoulis, C., Sioura, G., Kornezou, P., Vasiliadou, C., \& Tsioptsias, C. (2011). Contribution of okra extracts to the stability and rheology of oil-in-water emulsions. Food Hydrocolloids, 25(5), 991-999. http://dx.doi.org/10.1016/j.foodhyd.2010.09.014.

Gero, L. (2010). Type 1 diabetes mellitus: pathogenesis, symptoms and therapy. Orvosi Hetilap, 151(13), 533-539. http://dx.doi.org/10.1556/ oh.2010.28836. PMid:20304746.

Guo, W. L., Deng, J. C., Pan, Y. Y., Xu, J. X., Hong, J. L., Shi, F. F., Liu, G. L., Qian, M., Bai, W. D., Zhang, W., Liu, B., Zhang, Y. Y., Luo, P. J., Ni, L., Rao, P. F., \& Lv, X. C. (2020). Hypoglycemic and hypolipidemic activities of Grifola frondosa polysaccharides and their relationships with the modulation of intestinal microflora in diabetic mice induced by high-fat diet and streptozotocin. International Journal of Biological Macromolecules, 153, 1231-1240. http://dx.doi. org/10.1016/j.ijbiomac.2019.10.253. PMid:31759027. 
Guo, W. L., Shi, F. F., Li, L., Xu, J. X., Chen, M., Wu, L., Hong, J. L., Qian, M., Bai, W. D., Liu, B., Zhang, Y. Y., Ni, L., Rao, P. F., \& Lv, X. C. (2019). Preparation of a novel Grifola frondosa polysaccharidechromium (III) complex and its hypoglycemic and hypolipidemic activities in high fat diet and streptozotocin-induced diabetic mice. International Journal of Biological Macromolecules, 131, 81-88. http://dx.doi.org/10.1016/j.ijbiomac.2019.03.042. PMid:30851330.

Hu, R., Guo, W., Huang, Z., Li, L., Liu, B., \& Lv, X. (2018). Extracts of Ganoderma lucidum attenuate lipid metabolism and modulate gut microbiota in high-fat diet fed rats. Journal of Functional Foods, 46, 403-412. http://dx.doi.org/10.1016/j.jff.2018.05.020.

Huang, Y., Li, S. C., Hu, J., Ruan, H. B., Guo, H. M., Zhang, H. H., Wang, X., Pei, Y. F., Pan, Y., \& Fang, C. (2018). Gut microbiota profiling in Han Chinese with type 1 diabetes. Diabetes Research and Clinical Practice, 141, 256-263. http://dx.doi.org/10.1016/j. diabres.2018.04.032. PMid:29733871.

Lee, B. R., Lee, Y. P., Kim, D. W., Song, H. Y., Yoo, K. Y., Won, M. H., Kang, T. C., Lee, K. J., Kim, K. H., Joo, J. H., Ham, H. J., Hur, J. H., Cho, S. W., Han, K. H., Lee, K. S., Park, J., Eum, W. S., \& Choi, S. Y. (2010). Amelioration of streptozotocin-induced diabetes by Agrocybe chaxingu polysaccharide. Molecules and Cells, 29(4), 349-354. http://dx.doi.org/10.1007/s10059-0100044-9. PMid:20213314.

Li, L., Guo, W., Zhang, W., Xu, J., Qian, M., Bai, W., Zhang, Y., Rao, P., Ni, L., \& Lv, X. (2019). Grifola frondosa polysaccharides ameliorate lipid metabolic disorders and gut microbiota dysbiosis in high-fat diet fed rats. Food \& Function, 10(5), 2560-2572. http://dx.doi. org/10.1039/C9FO00075E. PMid:30994668.

Lv, X. C., Guo, W. L., Li, L., Yu, X. D., \& Liu, B. (2019). Polysaccharide peptides from Ganoderma lucidum ameliorate lipid metabolic disorders and gut microbiota dysbiosis in high-fat diet-fed rats. Journal of Functional Foods, 57, 48-58. http://dx.doi.org/10.1016/j. jff.2019.03.043.

O'Callaghan, S. (2017). Diagnosing diabetes mellitus. Physician Assistant Clinics, 2(1), 1-12. http://dx.doi.org/10.1016/j.cpha.2016.08.003.

Sánchez-Tapia, M., Tovar, A. R., \& Torres, N. (2019). Diet as regulator of gut microbiota and its role in health and disease. Archives of Medical Research, 50(5), 259-268. http://dx.doi.org/10.1016/j. arcmed.2019.09.004. PMid:31593850.

Shaw, J. E., Sicree, R. A., \& Zimmet, P. Z. (2010). Global estimates of the prevalence of diabetes for 2010 and 2030. Diabetes Research and Clinical Practice, 87(1), 4-14. http://dx.doi.org/10.1016/j. diabres.2009.10.007. PMid:19896746.

Sinha, R., Haileselassie, Y., Nguyen, L. P., Tropini, C., Wang, M., Becker, L. S., Sim, D., Jarr, K., Spear, E. T., Singh, G., Namkoong,
H., Bittinger, K., Fischbach, M. A., Sonnenburg, J. L., \& Habtezion, A. (2020). Dysbiosis-induced secondary bile acid deficiency promotes intestinal inflammation. Cell Host \& Microbe, 27(4), 659-670.e655. http://dx.doi.org/10.1016/j.chom.2020.01.021. PMid:32101703.

Tang, B. S., Lai, P. F., Wu, L., Li, Y. B., Shen, H. S., \& Chen, C. J. (2017). Recipe optimization and in vitro antioxidant properties of wheat bran dietary fiber chewable tablets. Food Science, 38(24), 171-176. http://dx.doi.org/10.7506/spkx1002-6630-201724027.

Waters, J. L., \& Ley, R. E. (2019). The human gut bacteria Christensenellaceae are widespread, heritable, and associated with health. BMC Biology, 17(1), 83. http://dx.doi.org/10.1186/s12915-019-0699-4. PMid:31660948.

Weng, M. J., Chen, J. C., Shen, H. S., Lai, P. F., \& Li, Y. B. (2017). A review on utilizations of okra extracts for food, medicine and environmental protection. Fujian Journal of Agricultural Sciences, 32(6), 685-690. http://dx.doi.org/10.19303/j.issn.1008-0384.2017.06.020.

Wu, L., Weng, M. J., Zheng, H. G., Lai, P. F., Tang, B. S., Chen, J. C., \& Li, Y. B. (2020). Hypoglycemic effect of okra aqueous extract on streptozotocin-induced diabetic rats. Food Science and Technology, 40(4), 972-978. http://dx.doi.org/10.1590/fst.28619.

Yuan, H., Shi, F., Meng, L., \& Wang, W. (2018). Effect of sea buckthorn protein on the intestinal microbial community in streptozotocin-induced diabetic mice. International Journal of Biological Macromolecules, 107(Pt A), 1168-1174. http://dx.doi.org/10.1016/j.ijbiomac.2017.09.090. PMid:28951300.

Yuan, Q., Lin, S., Fu, Y., Nie, X. R., Liu, W., Su, Y., Han, Q. H., Zhao, L., Zhang, Q., Lin, D. R., Qin, W., \& Wu, D. T. (2019). Effects of extraction methods on the physicochemical characteristics and biological activities of polysaccharides from okra (Abelmoschus esculentus). International Journal of Biological Macromolecules, 127, 178-186. http://dx.doi.org/10.1016/j.ijbiomac.2019.01.042. PMid:30639655.

Zhang, Y., Gu, Y., Ren, H., Wang, S., Zhong, H., Zhao, X., Ma, J., Gu, X., Xue, Y., Huang, S., Yang, J., Chen, L., Chen, G., Qu, S., Liang, J., Qin, L., Huang, Q., Peng, Y., Li, Q., Wang, X., Kong, P., Hou, G., Gao, M., Shi, Z., Li, X., Qiu, Y., Zou, Y., Yang, H., Wang, J., Xu, G., Lai, S., Li, J., Ning, G., \& Wang, W. (2020). Gut microbiome-related effects of berberine and probiotics on type 2 diabetes (the PREMOTE study). Nature Communications, 11(1), 5015. http://dx.doi.org/10.1038/ s41467-020-18414-8. PMid:33024120.

Zhou, W. B., Guo, R., Guo, W. L., Hong, J. L., Li, L., Ni, L., Sun, J. Y., Liu, B., Rao, P. F., \& Lv, X. C. (2019). Monascus yellow, red and orange pigments from red yeast rice ameliorate lipid metabolic disorders and gut microbiota dysbiosis in Wistar rats fed on a high-fat diet. Food \& Function, 10(2), 1073-1084. http://dx.doi.org/10.1039/ C8FO02192A. PMid:30720827. 